\title{
Correlation Between 25-Hydroxyvitamin D Level and Cardiac Diastolic Dysfunction in Chinese Adults with Early-Onset Type 2 Diabetes Mellitus: A Cross-Sectional Study
}

Lei Xiu

Xiao-ai Yao

Tao Jiang (1D

Department of Endocrinology, Beijing Shijitan Hospital, Capital Medical University, Beijing, 100038, People's Republic of China
Correspondence: Tao Jiang

Department of Endocrinology, Beijing Shijitan Hospital, Capital Medical

University, No. 10 Tieyilu, Yang Fang Dian,

Beijing, 100038, People's Republic of

China

Tel/Fax +86-10-63926692

Email jiangtao@bjsjth.cn
Purpose: Chinese adults with early-onset type 2 diabetes mellitus have impaired diastolic function. This study aims to analyse the association between serum vitamin D levels and cardiac diastolic dysfunction in Chinese adults with early-onset type 2 diabetes mellitus.

Patients and Methods: We enrolled Chinese adults with early-onset type 2 diabetes mellitus in this study. These patients were divided into two groups: those with diastolic dysfunction and those without diastolic dysfunction. We then compared the levels of serum 25-hydroxyvitamin $\mathrm{D}[25-(\mathrm{OH}) \mathrm{D}]$ between the two groups. The correlation between diastolic function and 25-(OH) $\mathrm{D}$ was evaluated by Pearson correlation analysis. Finally, binary logistic regression was used to analyse the relationship between the decrease in diastolic function and 25-(OH)D and other indexes in Chinese adults with early-onset type 2 diabetes mellitus.

Results: The level of 25-(OH)D in patients with early-onset type 2 diabetes mellitus complicated with cardiac diastolic dysfunction was significantly lower than that in patients without cardiac diastolic dysfunction $(\mathrm{P}<0.01)$. The degree of liver fibrosis in adult patients with early-onset type 2 diabetes mellitus complicated with diastolic dysfunction was significantly higher than that in adult patients without diastolic dysfunction $(\mathrm{P}<0.01)$. Moreover, decreased 25-(OH)D levels were associated with decreased diastolic function in adults with early-onset type 2 diabetes.

Conclusion: 25-(OH)-D was identified as an independent predictor of decreased diastolic function in adults with early-onset type 2 diabetes. The serum $25-(\mathrm{OH}) \mathrm{D}$ level in adults with early-onset type 2 diabetes was significantly reduced. 25-(OH)D influences the reduction in diastolic function in adults with early-onset type 2 diabetes and can be used as a predictor of decreased diastolic function in such patients.

Keywords: early-onset type 2 diabetes in adults, 25-hydroxyvitamin D, decreased diastolic function, non-alcoholic fatty liver disease

\section{Introduction}

The incidence rate of diabetes mellitus in China is increasing annually, especially among middle-aged and younger people. In 2010, the China Centers for Disease Control and Prevention (CDC) conducted a nationwide epidemiological survey, which showed that $5.7 \%$ of adults with diabetes in China were under 40 years old, and the proportion was increasing annually. ${ }^{1}$ At present, increasing attention has been given to adult early-onset type 2 diabetes mellitus (T2DM). Diabetes can be 
accompanied by a variety of chronic complications, including diabetic retinopathy, diabetic nephropathy, diabetic neuropathy and diabetic cardiomyopathy. Diabetic cardiomyopathy is a clinical disease diagnosed when ventricular dysfunction occurs in diabetic patients without coronary atherosclerosis and hypertension. The associated pathophysiological abnormalities include cardiac insulin resistance, glucotoxicity, oxidative stress, mitochondrial dysfunction, endoplasmic reticulum stress, impaired calcium homeostasis, phagocyte and autoantibody dysfunction, systemic and tissue renin-angiotensin-aldosterone system (RAAS) system activation and coronary microvascular dysfunction. $^{2}$ Previous studies have shown that vitamin D deficiency is associated with subclinical cardiac dysfunction and an increased risk of cardiovascular disease. ${ }^{3-5}$ However, there are few studies on the relationship between 25-hydroxyvitamin D [25-(OH)D] and T2DM, especially adult early-onset T2DM with cardiac insufficiency. This is the focus of our research. Notably, there is a significant correlation between diabetes mellitus and congestive heart failure caused by diastolic dysfunction in the absence of impaired systolic function, and this type of heart failure is closely related to abnormal left ventricular diastolic function. ${ }^{6}$ An early feature of diabetic cardiomyopathy is abnormal diastolic function. Therefore, diastolic function is often used as a predictor to assess the risk of diabetic cardiomyopathy in diabetic patients. ${ }^{2}$ Studies have shown that vitamin $\mathrm{D}$ receptors are present in islet $\beta$ cells and that vitamin $\mathrm{D}$ can promote the secretion and sensitivity of insulin. ${ }^{7,8}$ Moreover, 25-(OH)D is the main storage form of vitamin D in blood circulation. ${ }^{9}$ In clinical research, 25-(OH) $\mathrm{D}$ is the most commonly used active metabolite of vitamin D. ${ }^{10}$ In addition, the level of 25-(OH)D is stable and can be repeatedly measured. ${ }^{11,12}$ Therefore, we chose $25-(\mathrm{OH}) \mathrm{D}$ as the research object.

At present, most studies have focused on T2DM as a whole, and there are few studies on the specific group of adults with early-onset T2DM, especially clinical research on the risk of decreased diastolic function in adult patients with early-onset T2DM. Our research focuses on early-onset T2DM in adults. The main reasons are as follows: 1 . The onset age of early-onset T2DM in adults is younger, the incidence of cardiovascular diseases such as coronary heart disease is lower than that in elderly patients, and the occurrence of cardiac diastolic dysfunction is higher; ${ }^{1} 2$. As the onset of the disease occurs at a young age, preventing or delaying the occurrence of chronic complications, including diabetic cardiomyopathy, is the main goal of adult early-onset T2DM treatment. ${ }^{13}$ Therefore, it is of great clinical significance to study this specific group of adults with early-onset T2DM.

We found that a large number of adult patients with earlyonset T2DM had left ventricular diastolic dysfunction, and the 25-(OH)D level in these patients was significantly lower than that in patients without left ventricular diastolic dysfunction. Our study attempts to investigate the correlation between 25-(OH)D levels and left ventricular diastolic dysfunction in adult patients with early-onset T2DM.

\section{Patients and Methods}

\section{Patient Population}

Patients with early-onset T2DM (onset age $<40$ years), with a mean age of $33.5 \pm 6.4$ years old, who were diagnosed and treated in the Department of Endocrinology of Beijing Shijitan Hospital Affiliated with Capital Medical University from July 2017 to October 2020 were included. Among them, 178 patients were not complicated with cardiac diastolic dysfunction, and 102 patients were complicated with cardiac diastolic dysfunction. The inclusion criteria were as follows: patients with T2DM with an onset age of 40 years and younger who met the diagnostic criteria and classification criteria of the World Health Organization (WHO) established in $1999,{ }^{14}$ namely, symptoms of diabetes mellitus plus a casual plasma glucose concentration $\geq 11.1 \mathrm{mmol} / \mathrm{L}$ or fasting plasma glucose (FPG) level $\geq 7.0 \mathrm{mmol} / \mathrm{L}$ or 2-hour plasma glucose ( $2 \mathrm{hPG}$ ) level $\geq 11.1 \mathrm{mmol} / \mathrm{L}$ after a $75 \mathrm{~g}$ glucose load. If there are no symptoms of diabetes, two of the three tests mentioned above need to be carried out on different dates and show positive results for diabetes. The exclusion criteria were as follows: 1) acute and chronic infections, autoimmune diseases, coronary heart disease, rheumatic heart disease and other primary cardiovascular diseases; 2) immune system diseases, hyperparathyroidism, multiple myeloma, malignant tumour bone damage, bone metastases and serious liver and kidney diseases; 3) the use of hypoglycaemic drugs that significantly affect insulin sensitivity, such as thiazolidinediones and sodium glucose co-transporter-2 (SGLT2) inhibitors; and 4) the use of drugs that affect bone metabolism, such as glucocorticoids, calcium agents, calcitonin, active vitamin $\mathrm{D}$, common vitamin $\mathrm{D}$, and bisphosphonates.

We defined type 2 diabetes occurring before the age of 40 as early-onset T2DM in adults because most adults are diagnosed with T2DM after the age of 40 in China. ${ }^{15}$ In 
addition, the diabetes branch of the Chinese Medical Association recommends that adults over the age of 40 be screened for T2DM. ${ }^{16}$

\section{Methods}

Data from all the subjects were collected from a chart review, including height, weight, waist circumference, blood pressure and body mass index (BMI). After the patients fasted for 8 hours, blood samples were collected between 6:00 a.m. and 7:00 a.m. Blood glucose, glycated haemoglobin $(\mathrm{HbA} 1 \mathrm{C})$, insulin, serum calcium $\left(\mathrm{Ca}^{2+}\right)$, serum phosphorus $(\mathrm{P}), 25-(\mathrm{OH}) \mathrm{D}$ and triglyceride $(\mathrm{TG})$ levels were measured under fasting conditions. The 25$(\mathrm{OH}) \mathrm{D}$ level was measured with an automatic Roche ${ }^{\circledR} 601$ analyser, and the parathyroid hormone (PTH) level was measured with a chemiluminescence immunoassay (CLIA) on a Beckman ${ }^{\circledR}$ automatic analyser.

The levels of 25-(OH)-D in adults with early-onset T2DM were divided into four groups: $10(\mathrm{ng} / \mathrm{mL}), 10-20$ $(\mathrm{ng} / \mathrm{mL}), 20-30(\mathrm{ng} / \mathrm{mL})$ and $>30(\mathrm{ng} / \mathrm{mL})$. In binary logistic regression analysis, the continuous variable age was converted into categorical data as follows: $<20$ years old, 20-30 years old and 30-40 years old. The disease course was categorized as $<5$ years, $5-10$ years, $10-20$ years and $>$ 20 years. The blood glucose level was categorized as $<5$ $\mathrm{mmol} / \mathrm{L}, 5-10 \mathrm{mmol} / \mathrm{L}$ and $>10 \mathrm{mmol} / \mathrm{L}$. Similarly, other continuous variable data were converted to categorical data according to certain thresholds. The homeostasis model assessment of insulin resistance index (HOMA-IR) was calculated as fasting insulin (Fins) $\times \mathrm{FPG} / 22.5$.

The diagnosis of non-alcoholic fatty liver disease (NAFLD) was based on a method by Paul Angulo et al. ${ }^{17}$ T2DM patients with NAFLD were divided into groups according to the degree of liver fibrosis as follows: no liver fibrosis group, uncertain subgroup, and liver fibrosis subgroup. The NAFLD fibrosis score (NFS) was calculated as follows: $\mathrm{NFS}=1.675+0.037 \times$ age (years) $+0.094 \times \mathrm{BMI}$ $\left(\mathrm{kg} / \mathrm{m}^{2}\right)+1.13 \times \mathrm{IFG} /$ diabetes $($ yes $=1$, no $=0)+0.99 \times$ [aspartate aminotransferase (AST)/alanine aminotransferase (ALT) ratio] $-0.013 \times$ platelet $\left(\times 10^{9} / 1\right)-0.66 \times$ albumin $(\mathrm{g} /$ dl). ${ }^{17}$ According to the degree of liver fibrosis, T2DM patients with NAFLD were divided into the $<-1.455$ (no liver fibrosis group), - 1.455-0.676 (uncertain subgroup), and $>0.676$ (liver fibrosis subgroup) subgroups. The Fibrosis 4 (FIB-4) was calculated as age (years) $\times$ AST $[\mathrm{U} / \mathrm{L}] /($ platelets $\left.\left[10^{9} / \mathrm{L}\right] \times(\mathrm{ALT}[\mathrm{U} / \mathrm{L}])^{1 / 2}\right){ }^{18}$ The risk of advanced fibrosis was categorized on the basis of the FIB-4 value as low $(<1.30)$, intermediate $(1.30-2.67)$, or high $(>2.67) .{ }^{19}$
All subjects underwent an echocardiography examination (Toshiba Artida Color Doppler) by an experienced ultrasound physician. The average values of three measurements were obtained in the apical four-chamber view. The peak Doppler velocity and deceleration time (DT) of early diastolic (E wave) and end diastolic (A wave) filling were obtained. The presence or absence of diastolic dysfunction was ascertained by a professional ultrasound physician according to the following methods: ${ }^{20}$ impaired diastolic function, E/A $<$ 1 or DT $>240 \mathrm{~ms}$ (age $<55$ years) or $\mathrm{E} / \mathrm{A}<0.8$ and $\mathrm{DT}>$ $240 \mathrm{~ms}$ (age $\geq 55$ years); pseudonormal, E/A 1 to 1.5 , DT $>$ $240 \mathrm{~ms}$; and restrictive, DT $<160 \mathrm{~ms}$ and meeting one of the following conditions: $\mathrm{E} / \mathrm{A}>1.5$ or LA diameter $>5 \mathrm{~cm}$.

\section{Statistical Analysis}

SPSS 25.0 software was used for statistical analysis. The Kolmogorov-Smirnov test was used to test whether the distribution was normal. Normally distributed data are expressed as the mean \pm standard deviation $(\mathrm{x} \pm \mathrm{s})$. The comparison between the two groups was conducted by independent sample $t$ test. Chi square test was used for counting data. The correlation analysis of each index was performed by Pearson correlation analysis, and $\mathrm{P}<0.05$ was considered statistically significant. Nonnormally distributed data are expressed as the median and interquartile range [M (lower quartile, upper quartile)], and the comparison between the two groups was performed with the Mann-Whitney test. Binary logistic regression was used to analyse the risk factors for decreased diastolic function in adults with early-onset T2DM.

This study was approved by the ethics committee of Beijing Shijitan Hospital affiliated with Capital Medical University. The study was conducted according to the Declaration of Helsinki of the World Medical Association (ethical principles of medical research involving the human body). Written informed consent was received from participants prior to inclusion in the study.

\section{Results \\ Comparison of Basic Clinical Data, 25-(OH)D Levels and Biochemical Profiles}

Our study included 280 adult patients with early-onset T2DM who were diagnosed and treated in the Department of Endocrinology at Beijing Shijitan Hospital Affiliated with Capital Medical University from 2017 to 2020, including 178 patients without left ventricular diastolic dysfunction 
and 102 patients with left ventricular diastolic dysfunction.

The general condition and laboratory test results of the two groups are shown in Table 1. The age, course of disease, FIB-4 and NFS in the group with early-onset T2DM who were found to have diastolic dysfunction were significantly higher than these parameters in the group without diastolic dysfunction ( $\mathrm{P}$

Table I Basic Clinical Data and 25-(OH)D Levels of 280 Adult Patients with Early-Onset T2DM

\begin{tabular}{|c|c|c|c|}
\hline Item & $\begin{array}{l}\text { Without Decreased Diastolic } \\
\text { Function }\end{array}$ & $\begin{array}{c}\text { With Decreased Diastolic } \\
\text { Function }\end{array}$ & $p$ value \\
\hline $25-(\mathrm{OH}) \mathrm{D}(\mathrm{ng} / \mathrm{mL})$ & $17.79 \pm 5.37$ & $12.42 \pm 2.85$ & $0.002^{* *}$ \\
\hline$<10[N(\%)]$ & 27 (I5.2\%) & $24(23.5 \%)$ & \\
\hline $10 \sim 20[\mathrm{~N}(\%)]$ & $113(63.5 \%)$ & $70(68.6 \%)$ & \\
\hline $20 \sim 30[N(\%)]$ & 31 (I7.4\%) & $8(7.9 \%)$ & \\
\hline$>30[\mathrm{~N}(\%)]$ & 7 (3.9\%) & $0(0 \%)$ & \\
\hline Age (years) & $35.34 \pm 6.66$ & $41.24 \pm 3.31$ & $0.00 I^{* *}$ \\
\hline Diabetes duration (years) & $2.63 \pm 2.77$ & $7.20 \pm 4.95$ & $0.002^{* *}$ \\
\hline Waist circumference $(\mathrm{cm})$ & $95.58 \pm|3.1|$ & $96.72 \pm 9.50$ & 0.762 \\
\hline BMI $\left(\mathrm{kg} / \mathrm{m}^{2}\right)$ & $27.46 \pm 4.39$ & $26.98 \pm 3.66$ & 0.702 \\
\hline Systolic BP (mmHg) & $126.34 \pm 7.04$ & $127.06 \pm 7.86$ & 0.747 \\
\hline Diastolic BP (mmHg) & $77.69 \pm 6.16$ & $78.35 \pm 8.73$ & 0.757 \\
\hline Hypertension (\%) & II (6.2\%) & 7 (6.4\%) & 0.986 \\
\hline Coronary heart disease (\%) & $0(0 \%)$ & $0(0 \%)$ & \\
\hline Triglyceride (mmol/l) & $3.32 \pm 4.35$ & $2.97 \pm 2.04$ & 0.767 \\
\hline Glucose (mg/dl) & $8.54 \pm 3.54$ & $7.66 \pm 2.36$ & 0.363 \\
\hline Insulin $(\mu \mathrm{U} / \mathrm{mL})$ & $9.6(3.7-13.8)$ & $9.7(5.2-17.2)$ & 0.411 \\
\hline HOMA-IR & $1.0(0.9-1.7)$ & $1.5(1.2-1.7)$ & 0.166 \\
\hline $\mathrm{HbAIC}(\%)$ & $8.30 \pm 2.10$ & $8.24 \pm|.4|$ & 0.940 \\
\hline Calcium (mg/dl) & $2.26 \pm 0.09$ & $2.21 \pm 0.10$ & 0.078 \\
\hline Phosphate (mg/dl) & $1.30 \pm 0.17$ & $1.30 \pm 0.20$ & 0.986 \\
\hline Types of hypoglycemic drugs & $\begin{array}{l}\text { Insulin secretagogues, insulin, } \\
\text { Metformin, Alpha-glucosidase } \\
\text { Inhibitors, DPP-IV inhibitor }\end{array}$ & $\begin{array}{l}\text { Insulin secretagogues, insulin, } \\
\text { Metformin, Alpha-glucosidase } \\
\text { Inhibitors, DPP-IV inhibitor }\end{array}$ & \\
\hline NAFLD (\%) & $50 \%$ & $71 \%$ & 0.188 \\
\hline $\begin{array}{l}\text { NFS } \\
\qquad \begin{array}{l}<-1.455(\%) \\
-1.455-0.676(\%) \\
>0.676(\%)\end{array}\end{array}$ & $\begin{array}{c}-1.7| \pm| .22 \\
89(50 \%) \\
72(40 \%) \\
17(10 \%)\end{array}$ & $\begin{array}{c}-0.7 I \pm 0.76 \\
48(47 \%) \\
49(48 \%) \\
5(5 \%)\end{array}$ & $0.006 * *$ \\
\hline $\begin{array}{l}\text { FIB-4 } \\
\qquad \begin{array}{l}<1.30(\%) \\
1.30-2.67(\%) \\
>2.67(\%)\end{array}\end{array}$ & $\begin{array}{c}0.661 \pm 0.277 \\
166(93.3 \%) \\
12(6.7 \%) \\
0(0 \%)\end{array}$ & $\begin{array}{c}0.989 \pm 0.735 \\
92(90.2 \%) \\
5(4.9 \%) \\
5(4.9 \%)\end{array}$ & $0.030 *$ \\
\hline
\end{tabular}

Notes: Early-onset T2DM with decreased diastolic function compared with early-onset T2DM without decreased diastolic function; $* \mathrm{P}<0.05$, $* * \mathrm{P}<0.0 \mathrm{l}$.

Abbreviations: BMI, body mass index; BP, blood pressure; HbAIC, glycosylated haemoglobin; NAFLD, non-alcoholic fatty liver disease; HOMA-IR, homeostasis model assessment of insulin resistance index; 25-(OH)D, 25-hydroxyvitamin D; NFS, NAFLD fibrosis score; FIB-4, fibrosis-4. 
$<0.05)$. The level of $25-(\mathrm{OH}) \mathrm{D}$ in patients with left ventricular diastolic dysfunction was significantly lower than that in patients without left ventricular diastolic dysfunction $(\mathrm{P}<$ 0.01). No other statistically significant differences were observed between the groups, including waist circumference, BMI, TG, glucose, insulin, HOMA-IR, HbA1C, systolic blood pressure, diastolic blood pressure, prevalence of hypertension, prevalence of coronary heart disease, types of hypoglycemic drugs, calcium and phosphate.

\section{Comparison of Echocardiographic Parameters}

The E wave level in patients with early-onset T2DM with decreased left ventricular diastolic function was significantly lower than those in patients without left ventricular diastolic dysfunction $(\mathrm{P}<0.05)$. Furthermore, The E/A ratio, reflecting cardiac diastolic function, in patients with early-onset T2DM with decreased left ventricular diastolic function was significantly lower than those in patients without left ventricular diastolic dysfunction $(\mathrm{P}<0.001)$. No other statistically significant differences were observed between the groups, including the A wave, LVEF (left ventricular ejection fraction) and prevalence of left ventricular hypertrophy (Table 2).

\section{Correlation Analysis Between Left Ventricular Diastolic Function and Various Indexes}

$\mathrm{E} / \mathrm{A}$, an indicator of left ventricular diastolic dysfunction, was negatively correlated with $25-(\mathrm{OH}) \mathrm{D}(\mathrm{P}<0.01)$ and positively correlated with NFS, age and course of disease ( $\mathrm{P}$ $<0.01$ ) but not with blood glucose, HbA1C or TG (Table 3).

\section{Binary Logistic Regression Analysis of Risk Factors for Left Ventricular Diastolic Dysfunction}

Univariate logistic regression analysis was performed on 25-(OH)D, NFS, systolic blood pressure, diastolic blood pressure, glucose, HbA1C, NAFLD, diabetes duration, BMI, TG, albumin, insulin, and age. The results showed that $25-(\mathrm{OH}) \mathrm{D}(\mathrm{OR}=1.059, \mathrm{P}=$ $0.334)$, NFS $(\mathrm{OR}=2.677, \mathrm{P}=0.019)$, NAFLD $(\mathrm{OR}=$ $2.868, \mathrm{P}=0.117)$, duration $(\mathrm{OR}=1.363, \mathrm{P}=0.002)$ and age $(\mathrm{OR}=1.323, \mathrm{P}=0.004)$ should be entered in the binary multivariate logistic regression model. The results showed that 25-(OH)D $(\mathrm{OR}=1.936, \mathrm{P}=$ 0.042), NFS $(\mathrm{OR}=22.275, \mathrm{P}=0.043)$ and duration $(\mathrm{OR}=2.669, \mathrm{P}=0.017)$ were the main determinants of left ventricular diastolic dysfunction in adults with early-onset T2DM (Table 4).

Table 2 Comparison of Echocardiographic Parameters in 280 Adult Patients with Early-Onset T2DM

\begin{tabular}{|l|c|c|c|}
\hline Item & $\begin{array}{c}\text { Without Decreased Diastolic } \\
\text { Function }\end{array}$ & $\begin{array}{c}\text { With Decreased Diastolic } \\
\text { Function }\end{array}$ & $p$ value \\
\hline E wave $(\mathrm{cm} / \mathrm{s})$ & $89.17 \pm 20.46$ & $61.67 \pm 12.66$ & 0.047 \\
A wave $(\mathrm{cm} / \mathrm{s})$ & $60.42 \pm 12.38$ & $77.67 \pm 22.12$ & 0.085 \\
E/A ratio & $1.49 \pm 0.22$ & $0.81 \pm 0.09$ & $<0.001$ \\
LVEF & $63.38 \pm 10.61$ & $62.53 \pm 11.62$ & 0.798 \\
LV hypertrophy (\%) & $0(0 \%)$ & $0(0 \%)$ & \\
\hline
\end{tabular}

Note: Early-onset T2DM with decreased diastolic function compared with early-onset T2DM without decreased diastolic function. Abbreviations: LVEF, left ventricular ejection fraction; LV, left ventricle.

Table 3 Correlation Analysis Between Decreased Diastolic Function and Clinical Indicators in Patients with Early-Onset T2DM

\begin{tabular}{|l|c|c|c|c|c|c|c|}
\hline Item & Age & 25-(OH)D & NFS & Duration & Glucose & HbAIC & TG \\
\hline Diastolic function of heart & & & & & & & \\
$r$ & $0.468^{* *}$ & $-0.525^{* *}$ & $0.633^{* *}$ & $0.518^{* *}$ & -0.207 & 0.185 & -0.148 \\
$\quad P$ value & 0.004 & 0.002 & $<0.001$ & 0.001 & 0.219 & 0.462 & 0.397 \\
\hline
\end{tabular}

Notes: The correlation between the indexes and $E / A$ was analysed. $* * P<0.01$.

Abbreviations: 25-(OH)D, 25-hydroxyvitamin D; NFS, NAFLD fibrosis score; HbAIC, glycosylated haemoglobin; TG, triglyceride. 
Table 4 Binary Logistic Regression Analysis for Independent Associations of Decreased Left Ventricular Diastolic Function in EarlyOnset T2DM Patients

\begin{tabular}{|c|c|c|c|c|c|c|}
\hline \multirow[t]{2}{*}{ Covariate } & \multicolumn{3}{|c|}{ Univariate Analysis } & \multicolumn{3}{|c|}{ Multivariate Analysis } \\
\hline & OR & $95 \% \mathrm{Cl}$ & $P$ value & OR & $95 \% \mathrm{Cl}$ & $P$ value \\
\hline $25-(\mathrm{OH}) \mathrm{D}$ & 1.059 & $0.943-1.189$ & 0.334 & 1.936 & $|.024-3.66|$ & $0.042^{*}$ \\
\hline NFS & 2.677 & I.I78-6.080 & $0.019 *$ & 22.275 & I.10I-450.493 & $0.043^{*}$ \\
\hline Glucose & 0.910 & $0.744-1.113$ & 0.358 & & & \\
\hline HOMA-IR & 1.374 & $0.282-55.431$ & 0.308 & & & \\
\hline $\mathrm{HbAIC}$ & 0.982 & $0.620-1.553$ & 0.937 & & & \\
\hline NAFLD & 2.868 & $0.767-10.717$ & 0.117 & 0.02 & $0.000-1.311$ & 0.067 \\
\hline Duration & 1.363 & $1.122-1.656$ & $0.002 *$ & 2.669 & $1.195-5.962$ & $0.017^{*}$ \\
\hline BMI & 0.971 & $0.839-1.124$ & 0.695 & & & \\
\hline TGs & 0.973 & $0.814-1.162$ & 0.761 & & & \\
\hline Albumin & 0.922 & $0.773-1.099$ & 0.365 & & & \\
\hline Insulin & 0.961 & $0.873-1.057$ & 0.409 & & & \\
\hline Age & 1.323 & $1.095-1.598$ & $0.004 *$ & 1.614 & $0.92 I-2.830$ & 0.095 \\
\hline Systolic BP (mmHg) & 1.014 & $0.934-1.101$ & 0.740 & & & \\
\hline Diastolic BP (mmHg) & 1.014 & $0.932-1.103$ & 0.752 & & & \\
\hline Constant & & & & 0.000 & & $0.04 I^{*}$ \\
\hline
\end{tabular}

Notes: Binary logistic regression analysis, $* \mathrm{P}<0.05$ was statistically significant.

Abbreviations: 25-(OH)D, 25-hydroxyvitamin D; NFS, NAFLD fibrosis score; FIB-4, fibrosis-4; HOMA-IR, homeostasis model assessment of insulin resistance index; HbAIC, glycosylated haemoglobin; NAFLD, non-alcoholic fatty liver disease; BMI, body mass index; TG, triglyceride; BP, blood pressure.

\section{Discussion}

Our study shows that the serum 25-(OH)D level in patients with early-onset T2DM complicated with cardiac diastolic dysfunction is significantly lower than that in patients without cardiac diastolic dysfunction. Vitamin D is closely related to diabetic cardiomyopathy. Vitamin D can interact with the vitamin D receptor (VDR) and participate in gene regulation in various physiological processes, including mineral homeostasis, bone metabolism, immune regulation, and cell proliferation and differentiation. Moreover, the VDR is expressed in cardiomyocytes. VDR dysfunction may be involved in the development of cardiovascular disease, and the loss of VDR function may act as a feedback mechanism to trigger active vitamin D synthesis. Vitamin D can protect the heart through a receptor-dependent pathway. In addition, vitamin D may regulate cardiac function related to the PARP1/SIRT1/mTOR pathway and the Fas/FasL pathway. ${ }^{21,22}$ The occurrence of diabetic cardiomyopathy is related to an increase in cardiac peroxisome proliferator-activated receptor (PPAR) $y$ and a decrease in PPAR $\delta$. Vitamin D can directly stimulate the expression of PPAR $\delta$ and inhibit the expression of PPAR $\gamma$, which can lead to a decrease in adipogenesis and may alleviate diabetic cardiomyopathy. ${ }^{23,24}$ Vitamin D can improve cardiovascular disease through anti-inflammatory, antioxidant and antifibrotic activities, regulating advanced glycation end product signal transduction, the renin angiotensin system and cardiac metabolism. ${ }^{25}$ In addition, vitamin D levels affect the occurrence of cardiac autonomic neuropathy in patients with type 1 and type 2 diabetes mellitus. ${ }^{26}$ In conclusion, vitamin D plays an important role in the occurrence and development of diabetic cardiomyopathy. Vitamin D deficiency may increase the risk of diabetic cardiomyopathy.

Previous studies have shown that the prevalence of hepatic steatosis and fibrosis in patients with T2DM is $\operatorname{high}^{27}$ and that the NFS may be a predictor of cardiovascular disease. ${ }^{28}$ Our study found that the prevalence of NAFLD in early T2DM patients with left ventricular diastolic dysfunction was higher than that in T2DM patients without left ventricular diastolic dysfunction $(71 \%$ vs $50 \%)$. Moreover, NFS and FIB-4, the indexes reflecting the degree of NAFLD-related liver fibrosis, were significantly higher in adult patients with early-onset T2DM combined with left ventricular diastolic dysfunction than in those patients without left ventricular diastolic dysfunction. More importantly, the NFS score is an independent risk factor for left ventricular diastolic dysfunction in adult patients with early-onset T2DM. Our findings were consistent with the research of Lee et al, ${ }^{29}$ who found that liver fibrosis was associated with left ventricular diastolic dysfunction in patients with T2DM. The 
pathophysiological relationship between NAFLD and diastolic dysfunction in patients with T2DM may be related to the following mechanisms: the increase in ectopic fat and visceral fat deposition in NAFLD patients can increase the thickness of epicardial fat, thus affecting cardiac diastolic function. ${ }^{30,31}$ In addition, the increase in resting tension in myocardial cells, fibrosis and the deposition of myocardial collagen and advanced glycation end products (AGEs) in diabetic patients are important reasons for the decrease in ventricular diastolic function. ${ }^{32}$ The liver secretes hepatokines during the progression of NAFLD; this protein influences myocardial metabolism through autocrine, paracrine and endocrine signalling. ${ }^{33}$ Moreover, NAFLD increases the synthesis of procoagulant factors, inflammatory markers and adhesion molecules in the liver, which increase the risk of cardiovascular disease. $^{34}$

Diabetic cardiomyopathy is caused by insulin resistance in heart tissue, compensatory hyperinsulinaemia and hyperglycaemia. The occurrence of diabetic cardiomyopathy is not related to other cardiac risk factors, such as coronary heart disease (CAD) or hypertension. However, in our study, we found that the level of blood glucose in patients with early-onset T2DM combined with decreased diastolic function was not higher than that in patients without decreased diastolic function. The serum insulin level and HOMA-IR were higher than those in patients without decreased diastolic function, but the differences were not statistically significant. The reason for this result may be that although we excluded drugs that can significantly affect insulin resistance, such as thiazolidinediones and SGLT2 inhibitors, which may affect cardiac load, we could not completely exclude all hypoglycaemic drugs, including metformin and insulin, due to different conditions of patients, which could affect insulin resistance.

\section{Study Limitations}

There are still some problems to be solved in our research. First, our study is a retrospective study. Only adult patients with early-onset T2DM were included. The effects of 25$(\mathrm{OH}) \mathrm{D}$ and NAFLD on cardiac diastolic function were not compared with those in subjects with normal glucose tolerance. Second, the study is a current survey, the relationship between research factors and conclusions is exploratory, and the causal relationship needs to be further confirmed by prospective research. Third, this study is a retrospective study. We cannot stop the drugs that affect insulin secretion and insulin resistance in advance. We excluded the use of drugs that have a greater impact on insulin resistance, such as thiazolidinediones and SGLT2 inhibitors. However, due to the widespread use of metformin, insulin secretagogues and insulin in hospitalized patients, we cannot exclude the effects of these drugs on blood glucose and insulin resistance levels. Finally, this is a single-centre study from a Chinese hospital, so the results should be interpreted with caution when they are applied to different clinical settings.

\section{Conclusions}

Our cross-sectional study of adult patients with early-onset T2DM showed a significant correlation between 25-(OH) $\mathrm{D}$ and decreased diastolic function after adjusting for other known insulin resistance and cardiovascular risk factors. It is necessary to be aware of the occurrence of decreased diastolic function in adult patients with early-onset T2DM with insufficient 25-(OH)D levels. Our study showed that the age and disease duration of adult patients with earlyonset T2DM complicated with diastolic dysfunction were significantly higher than those without diastolic dysfunction, which suggests that age and course of disease are related to the occurrence of cardiac diastolic dysfunction in adult patients with early-onset T2DM. In addition, our study found that hepatic fibrosis was significantly associated with decreased diastolic function in adult patients with early-onset T2DM, which suggests that the NFS score may be an indicator for cardiac diastolic dysfunction in diabetic patients. These findings, combined with the current research on the pathogenesis of impaired diastolic function in patients with type 2 diabetes, will help to further study the pathogenesis and possible new targets of cardiac diastolic dysfunction in patients with T2DM.

\section{Data Sharing Statement}

The main data analysed in this study were included in the published article.

\section{Ethics Approval and Informed Consent}

This research study was conducted retrospectively from data obtained for clinical purposes. This study was approved by the ethics committee of Beijing Shijitan Hospital affiliated with Capital Medical University. All persons gave their informed consent prior to their inclusion in the study. 


\section{Consent for Publication}

All participants agreed to publication of the study.

\section{Funding}

This work was supported by the China Railway Corporation (grant number J2016Z029).

\section{Disclosure}

The author(s) declare no potential conflicts of interest with respect to the research, authorship, and/or publication of this article.

\section{References}

1. Huo X, Gao L, Guo L, et al. Risk of non-fatal cardiovascular diseases in early-onset versus late-onset type 2 diabetes in China: a cross-sectional study. Lancet Diabetes Endocrinol. 2016;4 (2):115-124. doi:10.1016/S2213-8587(15)00508-2

2. Jia G, Whaley-Connell A, Sowers JR. Diabetic cardiomyopathy: a hyperglycaemia- and insulin-resistance-induced heart disease. Diabetologia. 2018;61(1):21-28. doi:10.1007/s00125-017-4390-4

3. Pekkanen MP, Ukkola O, Hedberg P, et al. Serum 25-hydroxyvitamin D is associated with major cardiovascular risk factors and cardiac structure and function in patients with coronary artery disease. Nutr Metab Cardiovasc Dis. 2015;25(5):471-478. doi:10.1016/j.numecd.2015.02.005

4. Nolte K, Herrmann-Lingen C, Platschek L, et al. Vitamin $\mathrm{D}$ deficiency in patients with diastolic dysfunction or heart failure with preserved ejection fraction. ESC Heart Fail. 2019;6(2):262-270. doi:10.1002/ehf2.12413

5. Ozer PK, Emet S, Karaayvaz EB, et al. Silent myocardial dysfunction in vitamin D deficiency. Arch Med Sci Atheroscler Dis. 2020;5(1): e153-e162. doi:10.5114/amsad.2020.97110

6. Poornima IG, Parikh P, Shannon RP. Diabetic cardiomyopathy: the search for a unifying hypothesis. Circ Res. 2006;98(5):596-605. doi:10.1161/01.RES.0000207406.94146.c2

7. Yilmaz H, Kaya M, Sahin M, Delibasi T. Is vitamin D status a predictor glycaemic regulation and cardiac complication in type 2 diabetes mellitus patients? Diabetes Metab Syndr. 2012;6(1):28-31. doi:10.1016/j.dsx.2012.05.007

8. Gluba-Brzozka A, Franczyk B, Cialkowska-Rysz A, Olszewski R, Rysz J. Impact of vitamin D on the cardiovascular system in advanced chronic kidney disease (CKD) and dialysis patients. Nutrients. 2018;10(6). doi:10.3390/nu10060709

9. Samelson EJ, Kiel DP, Broe KE, et al. Metacarpal cortical area and risk of coronary heart disease: the Framingham Study. Am $J$ Epidemiol. 2004;159(6):589-595. doi:10.1093/aje/kwh080

10. Wang TJ. Vitamin D and cardiovascular disease. Annu Rev Med. 2016;67(1):261-272. doi:10.1146/annurev-med-051214-025146

11. Rosen CJ, Abrams SA, Aloia JF, et al. IOM committee members respond to Endocrine Society vitamin D guideline. J Clin Endocrinol Metab. 2012;97(4):1146-1152. doi:10.1210/jc.2011-2218

12. Holick MF, Binkley NC, Bischoff-Ferrari HA, et al. Evaluation, treatment, and prevention of vitamin D deficiency: an Endocrine Society clinical practice guideline. J Clin Endocrinol Metab. 2011;96(7):1911-1930. doi:10.1210/jc.2011-0385

13. Hillier TA, Pedula KL. Complications in young adults with early-onset type 2 diabetes: losing the relative protection of youth. Diabetes Care. 2003;26(11):2999-3005. doi:10.2337/diacare.26.11.2999

14. Mayfield J. Diagnosis and classification of diabetes mellitus: new criteria. Am Fam Physician. 1998;58(6):1355-1362, 1369-1370.
15. Kong X, Xing X, Zhang X, Hong J, Yang W. Early-onset type 2 diabetes is associated with genetic variants of beta-cell function in the Chinese Han population. Diabetes Metab Res Rev. 2020;36(2):e3214. doi:10.1002/dmrr.3214

16. Jia W, Weng J, Zhu D, et al. Standards of medical care for type 2 diabetes in China 2019. Diabetes Metab Res Rev. 2019;35(6):e3158. doi:10.1002/dmrr.3158

17. Angulo P, Hui JM, Marchesini G, et al. The NAFLD fibrosis score: a noninvasive system that identifies liver fibrosis in patients with NAFLD. Hepatology. 2007;45(4):846-854. doi:10.1002/ hep. 21496

18. Anstee QM, Lawitz EJ, Alkhouri N, et al. Noninvasive tests accurately identify advanced fibrosis due to NASH: baseline data from the STELLAR Trials. Hepatology. 2019;70(5):1521-1530. doi:10.1002/ hep.30842

19. McPherson S, Stewart SF, Henderson E, Burt AD, Day CP. Simple non-invasive fibrosis scoring systems can reliably exclude advanced fibrosis in patients with non-alcoholic fatty liver disease. Gut. 2010;59(9):1265-1269. doi:10.1136/gut.2010.216077

20. Lubien E, DeMaria A, Krishnaswamy P, et al. Utility of B-natriuretic peptide in detecting diastolic dysfunction: comparison with Doppler velocity recordings. Circulation. 2002;105(5):595-601. doi:10.1161/ hc0502.103010

21. Qu H, Lin K, Wang H, et al. 1,25(OH)2D3 improves cardiac dysfunction, hypertrophy, and fibrosis through PARP1/SIRT1/ mTOR-related mechanisms in type 1 diabetes. Mol Nutr Food Res. 2017;61(5):5. doi:10.1002/mnfr.201600338

22. Zeng $\mathrm{X}$, Yu $\mathrm{X}$, Xiao $\mathrm{S}$, Yao $\mathrm{H}$, Zhu J. Effects of 1,25-dihydroxyvitamin D3 on pathological changes in rats with diabetic cardiomyopathy. Lipids Health Dis. 2017;16(1):109. doi:10.1186/s12944-017-0498-2

23. Lee TI, Kao YH, Chen YC, Huang JH, Hsiao FC, Chen YJ. Peroxisome proliferator-activated receptors modulate cardiac dysfunction in diabetic cardiomyopathy. Diabetes Res Clin Pract. 2013;100(3):330-339. doi:10.1016/j.diabres.2013.01.008

24. Wang YX. PPARs: diverse regulators in energy metabolism and metabolic diseases. Cell Res. 2010;20(2):124-137. doi:10.1038/ cr.2010.13

25. Lee TW, Lee TI, Chang CJ, et al. Potential of vitamin D in treating diabetic cardiomyopathy. Nutr Res. 2015;35(4):269-279. doi:10.1016/j.nutres.2015.02.005

26. Hansen CS, Fleischer J, Vistisen D, Ridderstrale M, Jensen JS, Jorgensen ME. High and low vitamin D level is associated with cardiovascular autonomic neuropathy in people with Type 1 and Type 2 diabetes. Diabet Med. 2017;34(3):364-371. doi:10.1111/ dme. 13269

27. Ciardullo S, Monti T, Perseghin G. High prevalence of advanced liver fibrosis assessed by transient elastography among U.S. adults with type 2 diabetes. Diabetes Care. 2021;44(2):519-525. doi:10.2337/ dc20-1778

28. Ciardullo S, Sala I, Perseghin G. Screening strategies for nonalcoholic fatty liver disease in type 2 diabetes: insights from NHANES 2005-2016. Diabetes Res Clin Pract. 2020;167:108358. doi:10.1016/ j.diabres.2020.108358

29. Lee H, Kim G, Choi YJ, et al. Association between non-alcoholic steatohepatitis and left ventricular diastolic dysfunction in type 2 diabetes mellitus. Diabetes Metab J. 2020;44(2):267-276. doi:10.4093/dmj.2019.0001

30. Levelt E, Pavlides M, Banerjee R, et al. Ectopic and visceral fat deposition in lean and obese patients with type 2 diabetes. J Am Coll Cardiol. 2016;68(1):53-63. doi:10.1016/j.jacc.2016.03.597

31. Petta S, Argano C, Colomba D, et al. Epicardial fat, cardiac geometry and cardiac function in patients with non-alcoholic fatty liver disease: association with the severity of liver disease. J Hepatol. 2015;62 (4):928-933. doi:10.1016/j.jhep.2014.11.030 
32. van Heerebeek L, Hamdani N, Handoko ML, et al. Diastolic stiffness of the failing diabetic heart: importance of fibrosis, advanced glycation end products, and myocyte resting tension. Circulation. 2008;117(1):43-51. doi:10.1161/CIRCULATIONAHA.107.728550

33. Meex RCR, Watt MJ. Hepatokines: linking nonalcoholic fatty liver disease and insulin resistance. Nat Rev Endocrinol. 2017;13(9):509-520. doi:10.1038/nrendo.2017.56
34. Adams LA, Anstee QM, Tilg H, Targher G. Non-alcoholic fatty liver disease and its relationship with cardiovascular disease and other extrahepatic diseases. Gut. 2017;66(6):1138-1153. doi:10.1136/gutjnl-2017313884

Diabetes, Metabolic Syndrome and Obesity: Targets and Therapy

\section{Publish your work in this journal}

Diabetes, Metabolic Syndrome and Obesity: Targets and Therapy is an international, peer-reviewed open-access journal committed to the rapid publication of the latest laboratory and clinical findings in the fields of diabetes, metabolic syndrome and obesity research. Original research, review, case reports, hypothesis formation, expert opinion and commentaries are all considered for publication. The manuscript management system is completely online and includes a very quick and fair peer-review system, which is all easy to use. Visit http://www.dovepress.com/testimonials.php to read real quotes from published authors.

Submit your manuscript here: https://www.dovepress.com/diabetes-metabolic-syndrome-and-obesity-targets-and-therapy-journal 